\title{
SIMULAÇÃO FÍSICA A FRIO EM ESCALA LABORATORIAL DA DENSIFICAÇÃO DE UMA MISTURA DE CARVÕES VIA VIBRAÇÃO MECÂNICA*
}

\section{Resumo}

Leandro Miranda Nascimento ${ }^{1}$ Paulo Santos Assis² Ronaldo Santos Sampaio ${ }^{3}$

A maior parte da produção mundial de aço passa pela conversão de minério de ferro em grandes altos-fornos, tendo o coque como uma das matérias-primas. Por um lado, boas propriedades mecânicas a quente do coque, como o CSR e CRI, são necessárias para que o consumo de coque seja o mínimo, pois é também, junto com os óxidos de ferro, um dos maiores componentes dos custos variáveis do ferro gusa. As misturas dos vários tipos de carvões minerais utilizados exigem a participação de uma fração Hard, a mais cara e com maior poder de coqueificação, e de uma fração Soft, mais barata e inerte. Logo, quanto maior for a participação do Hard mais caro será a mistura a ser coqueificada. Uma maneira de aumentar a fração de Soft na mistura de carvões, reduzindo o custo da mistura, e ainda assim obter um coque com boa qualidade é aumentar a densidade a granel da mistura. Para isso alguns métodos já são empregados com sucesso, como o Stamp Charging e a Briquetagem Parcial. Este artigo apresenta um novo método, que consiste na densificação da mistura de carvões dentro do forno via dispositivos de vibração. Foram realizadas simulações físicas a frio e em escala reduzida para avaliar o potencial de aumento de densidade a granel da técnica. Os resultados mostram um ganho de aumento de $30 \%$ da densidade a granel da mistura.

Palavras-chave: Coqueria; Simulação física; Siderurgia; Vibração; Densificação.

\section{PHYSICAL SIMULATION BY COLD AND IN LAB SCALE OF A COAL MIX DENSIFICATION BY VIBRATING}

\begin{abstract}
The Blast furnace technology represents globally more than $95 \%$ of all primary iron source to produce steel. The Blast-furnace is a counter current porous media where coke particles play the major role as porous media, energy source and reducer for the iron oxides. The coke is produced basically from two sources of metallurgical coals, the "hard" coal with coking properties (swelling) and another "soft", inert and cheaper that together produce the desirable coke. The coke with high CRS and low CRI ensure the high productivity and low fuel rate at the blast furnace. The coke mix (hard + soft) increases its costs the higher it is the fraction of the hard coal, a more expensive coal. However, if the bulk density of the blend (hard + soft) is increased it is possible to increase the fraction of poor coal, soft, in the blend. By this way coke oven productivity increase and the coke production cost is reduced keeping the same coke's quality level. Stamping Charging and Partial Briquetting are methods that exemplify this technique. This article introduces a new method to improve the coal mix bulk density by a vibration technique with patent pending. Some physical simulation in cold and reduced scale has been made to estimate the increase potential of density of the blend. The lab results presented up to $30 \%$ in the bulk density enhancement.

Keywords: Coke oven; Physical simulation; Iromaking; Vibration; Densification.

1 Engenheiro Metalúrgico, Aluno de Mestrado, REDEMAT, Universidade Federal de Ouro Preto, Ouro Preto, Minas Gerais, Brasil.

2 Engenheiro Metalúrgico, Professor Doutor, Professor Titular, DEMET/REDEMAT, Universidade Federal de Ouro Preto, Ouro Preto, Minas Gerais, Brasil

3 Engenheiro Metalúrgico, Doutor, CEO, RS Consultants LTDA, Belo Horizonte, Minas Gerais, Brasil.

4 Engenheiro Metalúrgico, Mestre, Consultor, Gerdau Ouro Branco, Ouro Branco, Minas Gerais, Brasil.
\end{abstract}




\section{INTRODUÇÃO}

O coque é o agente termoredutor nos altos-fornos e responde pela a produção de mais de $95 \%$ de todo o ferro primário produzido no mundo atualmente. Simplificando, o coque é o produto da coqueificação de misturas de vários tipos de carvões minerais onde dois tipos de famílias de carvões são necessários. Uma das famílias é dos Hard Coking Coals (HCC), onde há capacidade de se atingir expansões plásticas, entra como a responsável pela "cola" para a formação do corpo poroso, denso e homogêneo, denominado coque. De acordo com a norma ASTM [1] (ASTM D388), ilustrada na tabela I.I, nessa categoria se enquadram os carvões betuminosos de baixo, médio e alto volátil. A outra família é dos carvões soft, que entram como redutores de custo da mistura. Nessa categoria se encontram os lignitos e carvões sub-betuminosos.

Tabela I.I: Classificação ASTM dos carvões por classe:

\begin{tabular}{|c|c|c|c|c|c|c|c|c|}
\hline \multirow[t]{2}{*}{ Classe } & \multirow[t]{2}{*}{ Grupo } & \multicolumn{2}{|c|}{$\begin{array}{c}\text { Carbon } \\
\text { o Fixo } \\
\%\end{array}$} & \multicolumn{2}{|c|}{$\begin{array}{c}\text { Materia } \\
\text { I Volátil } \\
\%\end{array}$} & \multicolumn{2}{|c|}{$\begin{array}{c}\text { Poder } \\
\text { Calorífico } \\
\text { (BTU/libra) }\end{array}$} & \multirow{2}{*}{$\begin{array}{c}\text { Característica } \\
\text { s } \\
\text { aglomerantes }\end{array}$} \\
\hline & & $\geq$ & $<$ & $\geq$ & $<$ & $\geq$ & $<$ & \\
\hline \multirow[t]{3}{*}{ I-Antracito } & $\begin{array}{l}\text { 1. meta } \\
\text { antracito }\end{array}$ & 98 & - & & 2 & - & - & \multirow{3}{*}{$\begin{array}{c}\text { Não } \\
\text { Aglomerante }\end{array}$} \\
\hline & 2. antracito & 92 & 98 & 2 & 8 & - & - & \\
\hline & $\begin{array}{l}\text { 3. semi- } \\
\text { antracito }\end{array}$ & 86 & 92 & 8 & 14 & - & - & \\
\hline \multirow[t]{5}{*}{$\begin{array}{l}\text { Il - Carvão } \\
\text { Betuminos } \\
\text { o }\end{array}$} & 1. baixo volátil & 78 & 88 & 14 & 22 & - & - & \multirow{5}{*}{ Aglomerante } \\
\hline & $\begin{array}{l}\text { 2. médio } \\
\text { volátil }\end{array}$ & 69 & 78 & 22 & 31 & - & - & \\
\hline & 3. alto volátil $A$ & - & 69 & 31 & - & 14.000 & - & \\
\hline & 4. alto volátil $B$ & - & - & - & - & 13.00 & $\begin{array}{c}14.00 \\
0\end{array}$ & \\
\hline & $\begin{array}{l}\text { 5. alto volátil } \\
\text { C }\end{array}$ & - & - & - & - & 11.500 & $\begin{array}{c}13.00 \\
0\end{array}$ & \\
\hline \multirow[t]{3}{*}{$\begin{array}{l}\text { III - Sub- } \\
\text { betuminos } \\
0\end{array}$} & 1. carvão A & - & - & - & - & 10.500 & $\begin{array}{c}11.50 \\
0\end{array}$ & \multirow{5}{*}{$\begin{array}{c}\text { Não } \\
\text { Aglomerante }\end{array}$} \\
\hline & 2. carvão B & - & - & - & - & 9.500 & $\begin{array}{c}10.50 \\
0\end{array}$ & \\
\hline & 3. carvão $C$ & - & - & - & - & 8.300 & 9.500 & \\
\hline \multirow[t]{2}{*}{ IV - Lignita } & 1. lignita $A$ & - & - & - & - & 8.300 & 8.300 & \\
\hline & 2. lignita $B$ & - & - & - & - & - & 6.300 & \\
\hline
\end{tabular}

No alto-forno, o coque tem as funções de fonte de energia, capacidade de redução dos óxidos de ferro e elemento estruturante do leito poroso necessário ao processo. Devido às condições de reatividade química, elevada temperatura e pressão nesse reator, as propriedades a quente do coque são de fundamental importância. O CSR 
e o CRI são dois dos principais indicadores de qualidade do coque a quente e são descritos pelas normas ISO 18894/2006 e ASTM D538/2014, entre outras.

Na coqueificação, o coque é o produto do aquecimento de uma mistura constituída de carvão mineral até temperaturas da ordem de $1200^{\circ} \mathrm{C}$ durante um período que pode ser superior a 20 horas de processo. Esse aquecimento ocorre das extremidades dos fornos para o centro da mistura e causa diversas transformações físicas, químicas e metalúrgicas. A mistura de carvões passa por transformações como perda de umidade, contração inicial, expansão e formação de gases que, ao final, dão origem ao coque.

Alguns métodos são utilizados para definir as misturas de carvões de modo a garantir uma relação entre os componentes mineralógicos e as propriedades metalúrgicas do carvão -tal como fluidez e dilatação - que seja capaz de produzir um coque com parâmetros adequados de CSR e CRI. Segundo Silva [2], os métodos mais conhecidos são o do Schapiro e Gray e o diagrama de MOF (Miyazu et all).

O carvão alimentado na coqueria é um material cominuído e poroso, que apresenta baixos valores de densidade a granel. Reduzindo-se os espaços vazios, a densidade a granel seria aumentada, alterando as forças que iram atuar no processo de coqueificação, e fazendo com que, por exemplo, uma mistura de menor poder coqueificante apresentasse um valor de pressão de coqueificação superior ao valor de uma mistura não densificada. Tal efeito gera uma alteração no diagrama MOF, que implica na possibilidade de utilizar uma maior fração de carvões soft para obter um coque de qualidade tão boa quanto, ou em alguns casos até melhor, do coque obtido por uma mistura com maior fração de carvões $H C C$ pela forma convencional

Amorim [3] descreveu quais métodos de aumento de densidade que podem ser utilizados em cada tipo de coqueria, reforçando que em maior ou menor grau todos representam significativa redução do custo devido à diferença dos custos dos carvões soft e hard. Os principais métodos são a Briquetagem Parcial e o Stamp Charging. O primeiro é aplicado em coquerias convencionais ou não e consiste em fazer briquetes com a mistura de carvões. Esses briquetes são adicionados ao restante da carga na proporção de até 30\%. Como o briquete tem densidade superior a densidade média da mistura a enfornada, a densidade a granel da carga aumenta. Segundo Leibrock [4], o aumento por briquetagem parcial na densidade pode chegar a $15 \%$ em relação ao valor original da mistura que é da ordem de 650 $\mathrm{kg} / \mathrm{m}^{3}$. Já o Stamp Charging é aplicado somente em coquerias non-recovery/heat recovery e consiste em uma prensagem da carga anterior ao enfornamento. A mistura de carvões é prensada e enfornada de forma semelhante a um bolo, realizada por um carro alimentador que se situa na lateral da coqueria. Sua aplicação assim como uma lista das unidades industriais que dispõe de tal tecnologia foi recentemente descrita por Madias [5]. Tal processo pode atingir densidades a granel superiores a $1000 \mathrm{~kg} / \mathrm{m}^{3}$, de acordo com Leibrock [4]. Por possuírem grandes estruturas acopladas ao processo de coqueria ambos os métodos apresentam um alto custo de instalação, sendo o Stamp Charging o mais oneroso deles. Apesar disso, o Stamp Charging também é o método mais eficiente no aumento da densidade da carga enfornada.

O presente trabalho é parte do desenvolvimento de uma nova metodologia que visa permitir o adensamento da mistura de carvões na coqueria por meio de um mecanismo de vibração, assim como o utilizado na engenharia civil para adensar o concreto e garantir propriedades mecânicas mais adequadas. Foi estudado se a vibração poderia aumentar a densidade da mistura de carvões, concluindo-se que tal efeito pode ter uma implicação positiva tanto na redução do custo do carvão, por 
substituição de carvões HCC por carvões soft quanto na produtividade do processo, por permitir o aumento da densidade de carga enfornada. Esse processo foi objeto de patente por Assis [6].

\section{1 - Objetivo}

O objetivo desse trabalho é realizar uma simulação a frio do procedimento de alimentação da mistura de carvões em um modelo de acrílico com escala reduzida de uma coqueria real e avaliar se o uso de um vibrador seria capaz de aumentar a densidade da mistura de carvões, gerando uma redução de custo do processo e aumento da produtividade.

\section{MATERIAIS E MÉTODOS}

Visando identificar o potencial da técnica de vibração na compactação, e, consequentemente densificação, das misturas de carvões adotou-se uma metodologia para realizar a simulação física do processo de alimentação combinada com vibração em escala laboratorial.

Inicialmente, foi necessário estabelecer um critério ou uma metodologia para a construção de um protótipo que trabalhasse em escala reduzida com relação a um forno real de coqueria. Em fluidos, critérios como número de Euller, número de Reynolds, por exemplo, são bastante usais e conhecidos e devem ser utilizados para determinação das relações geométricas entre o protótipo e o sistema a ser estudado conforme as principais forças envolvidas nesse sistema. Contudo, as misturas de carvões são materiais fragmentados e apresentam comportamentos muito mais complexos do que os fluidos, conforme observado por Silva [7] esses materiais podem ora se comportar como sólido, ora como líquido e ora como gases, sendo então de grande dificuldade a descrição do comportamento desse material por equações. Por outro lado, Silva [7] descreve a formação de arco como um dos problemas mais relevantes no escoamento de material segmentado, já que acaba por estagnar e impedir o fluxo de material. Esse fenômeno não ocorre desde que a dimensão mínima da geometria estudada seja oito vezes maior que o tamanho máximo de partícula. No caso de misturas de carvões o tamanho máximo de partícula é de, em média, de $4 \mathrm{~mm}$, e a dimensão mínima do forno é sua largura. Portanto, um protótipo trabalhando com uma mistura real de coqueria atenderia ao critério de não formação de arco desde que tivesse dimensão de largura superior a $32 \mathrm{~mm}$.

Um ponto fundamental refere-se à maneira pela qual poderiam ser realizadas vibrações nos ensaios reproduzindo-as de modo similar no modelo real. As dimensões e as condições nas quais o processo de alimentação na coqueria convencional ocorre deixam clara a dificuldade de acesso à carga. No entanto, a maneira aparentemente mais viável de prover contato de um vibrador industrial de grandes dimensões com a carga de carvão já enfornada parece ser via boca de carga, que são quatro orifícios, com diâmetro aproximado ao da largura do forno, que se encontram na parte superior dos fornos de coqueria. Percebeu-se, então, que para estudos iniciais seria possível, por simetria, realizar a simulação de 1/4 do forno sendo vibrado utilizando um vibrador introduzido na mesma região de alimentação.

Sendo assim, um acrílico com as dimensões de $481 \mathrm{~mm}$ de altura, $281 \mathrm{~mm}$ de comprimento e $35 \mathrm{~mm}$ de largura média com conicidade de $2 \mathrm{~mm}$, foi construído em réplica de 1:13 da coqueria existente na Gerdau Açominas. 
Com a utilização de vibrador de imersão para concreto portátil, usual do setor de construção civil, foram realizados repetidos testes no modelo em acrílico. $O$ equipamento tem como parâmetros a frequência nominal de $12500 \mathrm{vpm}$, amplitude de $8 \mathrm{~mm}$, potência de $1,5 \mathrm{~kW}$ e diâmetro de ponta de $28 \mathrm{~mm}$. Tais testes consistiram em estimar a densidade a granel de uma mistura de carvões sem vibração e comparar com a densidade a granel da mistura alimentada utilizando um procedimento de vibração.

O carvão utilizado foi uma mistura padrão de coqueria convencional. Sua umidade foi de $7 \%$ e sua granulometria é apresentada no gráfico da figura 1 :

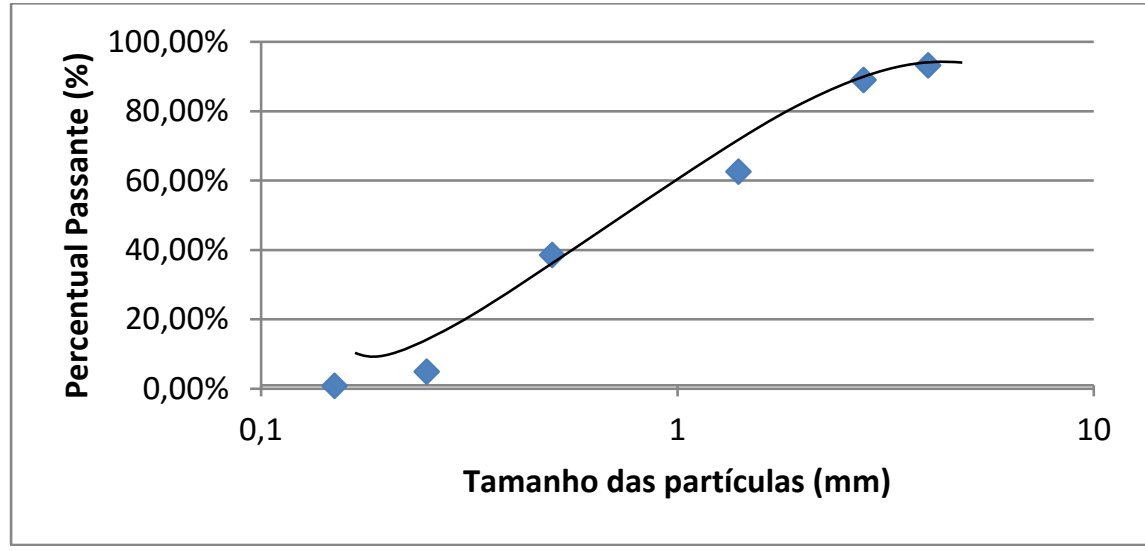

Figura 1: Distribuição granulométrica do carvão utilizado.

A caixa acrílica foi alimentada com a mistura de carvões a ser estudada na mesma posição de alimentação do processo. A caixa foi então totalmente preenchida e pesada em uma balança previamente tarada com seu peso. O peso encontrado foi então dividido pelo volume do recipiente e a densidade a granel foi calculada com base nesses valores, de acordo com a equação (1). Em seguida foi realizado um processo similar, com a inclusão o procedimento de vibração da carga em algum momento da alimentação da mistura de carvões. Ao final, a caixa era novamente preenchida por completo e pesada. Uma nova densidade a granel foi encontrada e a variável resposta do processo é o percentual de aumento da densidade em relação ao processo sem vibração. Para evitar os efeitos de uma eventual alteração do material foram realizados medições periódicas da densidade a granel antes do processo com vibração daquele material.

$$
\rho_{\text {à granel }}=\frac{(\text { Peso recipeinte preenchido-Peso recipiente vazio })}{\text { Volume do recipiente }}
$$

Os parâmetros avaliados foram: o número de inserção do vibrador, o tempo de vibração por inserção e tempo total de vibração. Em ensaios onde foi feita apenas uma única inserção do vibrador realizou-se o processo com a caixa acrílica próxima à sua carga máxima em volume. Nos ensaios com duas ou mais inserções, o volume das misturas alimentadas entre as inserções eram divididas proporcionalmente entre as vibrações. Realizou-se ainda um ensaio em que a vibração ocorreu ao mesmo tempo da alimentação. A figura 2 aponta o nível do carvão no acrílico em cada teste: 


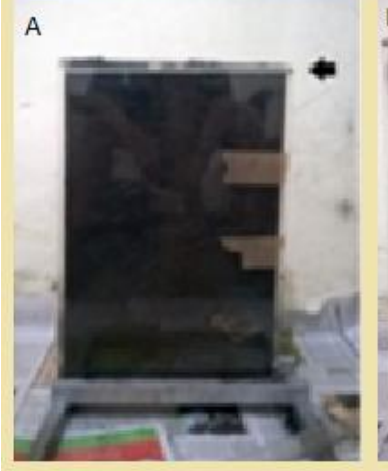

B

$B$

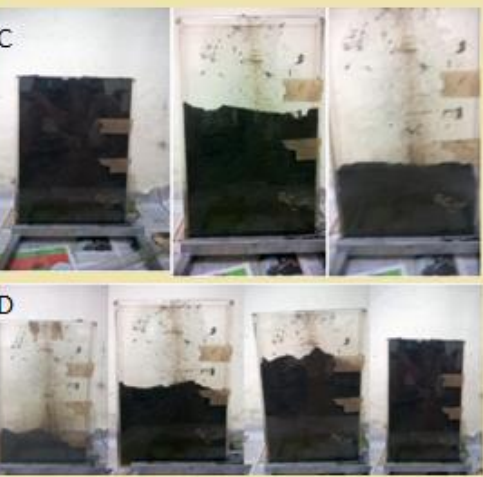

Figura 2: Nível de carvão no modelo de acrílico da coqueria no momento em que foi realizada as inserções em cada um dos ensaios. A) Uma inserção; B) Duas inserções; C) Três inserções; D) Quatro inserções.

\section{RESULTADOS E DISCUSSÃO}

A figura 3 apresenta o gráfico com os dados de variação da densidade a granel, em função do tempo em que a amostra foi submetida a vibração. Nesse primeiro cenário, foi realizada apenas uma inserção quando a caixa acrílica já se encontrava totalmente preenchida. Por meio do gráfico, pode-se observar que a técnica consegue alcançar um aumento densidade da ordem de $12 \%$, sem grandes variações com o aumento do tempo de vibração após o tempo de 20 segundos de vibração.

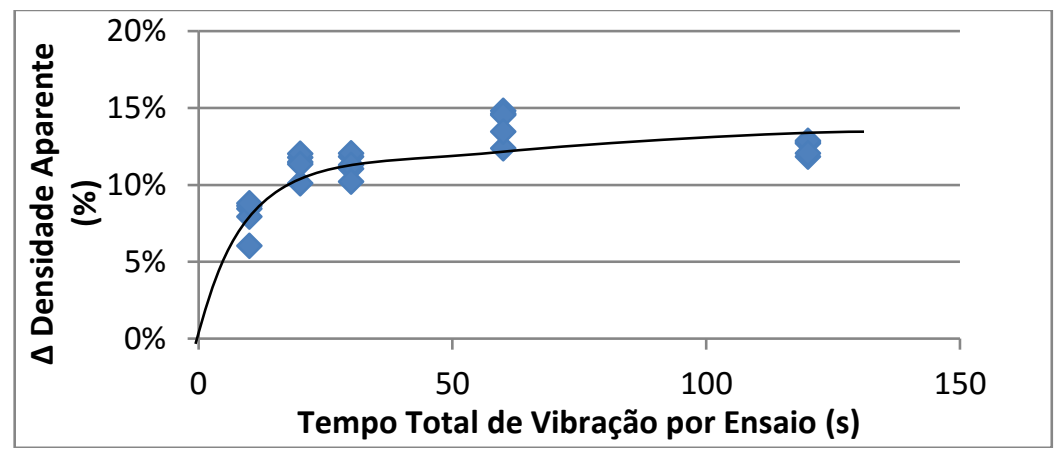

Figura 3: Aumento da densidade a granel em função do tempo de vibração com apenas uma inserção.

Entre os 60 e os 120 segundos, houve uma pequena redução no valor médio de variação da densidade, de 13,9\% para 12,4\%. Considerando que o desvio padrão dessa variável é de $1,1 \%$, pode-se mostrar por teste de hipótese entre as duas condições que a redução de densidade entre elas é inconclusiva, ou seja, estatisticamente não podemos dizer que o ganho em densidade a granel após a vibração de 60 segundos é menor que o ganho após 120 segundos.

Descrevendo qualitativamente a técnica, o vibrador é introduzido no acrílico já em funcionamento penetrando na mistura de carvões. Inicialmente percebe-se que o nível de material aumenta, projetando inclusive parte desse material para fora do acrílico à medida que o vibrador desce. Após poucos segundos, nota-se, visualmente, que o nível da mistura começa a diminuir em relação ao nível original. Tal fato é nítido e intenso durante os primeiros segundos da técnica, sendo 
suavizado após 20 segundos mantendo a mistura no mesmo nível ou com variações já pouco perceptíveis ao olho nu. Nesse momento, o espaço vazio é preenchido com uma nova carga de carvão visando restabelecer o nível de referência do acrílico para a sua pesagem. Essa etapa gera um erro de medição, já que o carvão alimentado está com uma densidade de carga diferente a do carvão alimentado anteriormente e que foi submetido ao processo de vibração. No entanto, esse é erro é menor que $2 \%$ do peso da mistura, e caso esse procedimento não fosse realizado, - erro devido da estimativa do volume que a mistura ocuparia seria mais significativo, pois o perfil da mistura de carvões após a vibração é bastante irregular sendo difícil estimar o volume.

Na figura 4, o gráfico mostra a variação da densidade a granel em função do tempo total de vibração em outro cenário. Nesse caso, o tempo de vibração em uma inserção de 30 segundos foi mantido constante, variando-se o número de inserções do vibrador na mistura por ensaio. No segundo cenário, ao contrário do anterior, fica nítido o aumento da variação percentual de densidade de carga com o aumento do tempo de exposição total do material à vibração. Sendo assim, é possível deduzir que só o tempo de vibração não é um fator determinante para o aumento da densidade, mas o modo como essa vibração é realizada afeta diretamente o resultado. É possível perceber ainda que, a medida que se aumenta o número de inserções é menos significativo o aumento na densidade. $O$ aumento de densidade chegou a atingir $30 \%$ em média quando quatro inserções foram realizadas no sistema.

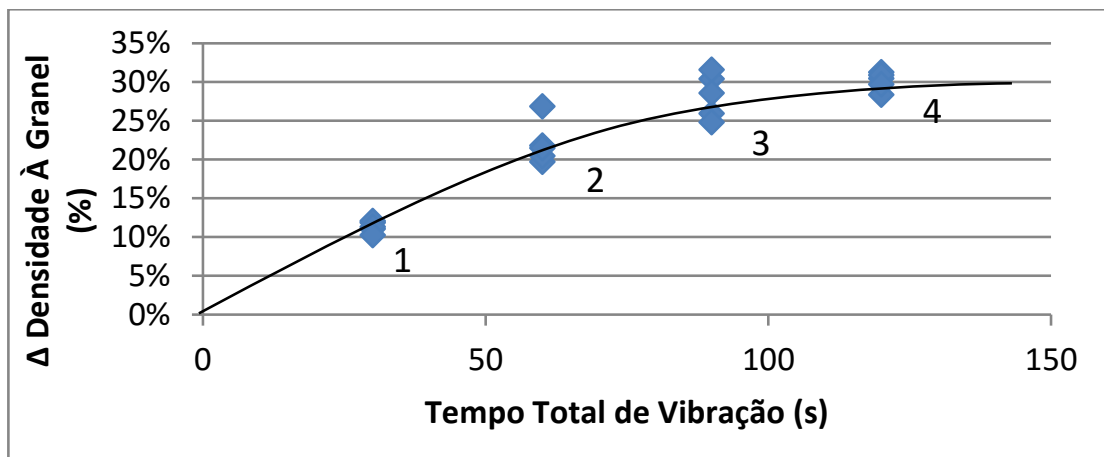

Figura 4: Gráfico da variação de densidade em função do tempo total de vibração para ensaios com diferentes números de inserções de 30 segundos cada.

Foi simulado ainda um terceiro cenário, mantendo uma vibração constante e concomitante à alimentação da mistura. A vibração foi feita no furo central e a alimentação foi realizada lateralmente ao vibrador. Na prática, seria necessária uma ampliação da boca de carga ou a construção de novas "bocas de carga" para realização desse procedimento, pois se somente o restante da área ocupada pelo vibrador na boca de carga fosse destinada a alimentação, essa taxa extremamente baixa de alimentação, e conseqüentemente, 0 tempo de processo seria extremamente elevado. Esse cenário teve um tempo médio de vibração de 78 segundos e alcançou um aumento de densidade média de $20 \%$, atingindo valores potencialmente menores do que os obtidos em vibrações constante de 30 segundos, com três e quatro inserções. Nesse cenário, o tempo varia, já que a alimentação de um material fragmentado sofre flutuações por agarramento lateral na saída do funil. A figura 5 mostra 0 aumento percentual do cenário de vibração constante, comparado aos outros dois cenários: 


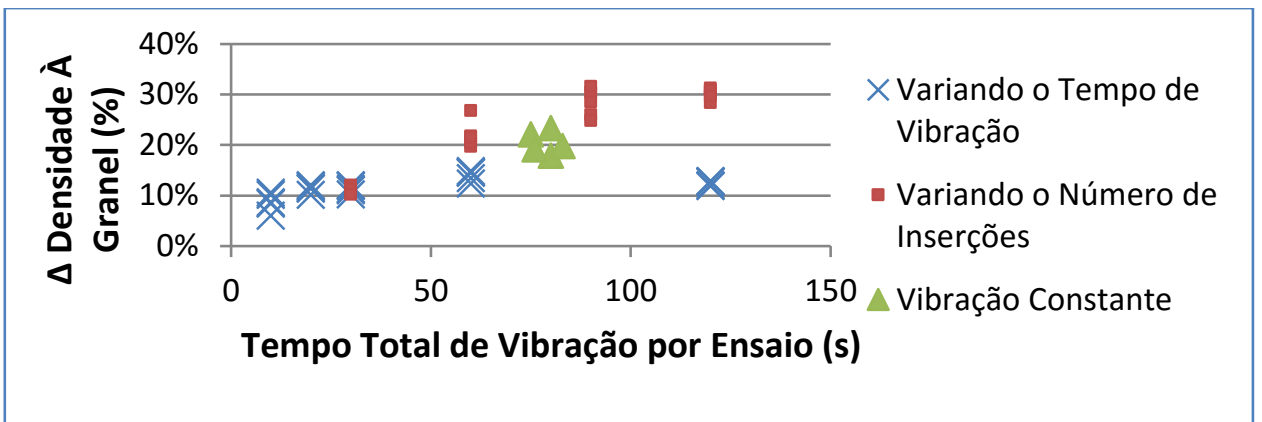

Figura 5: Comparativo correlacionando o tempo total de vibração e a variação da densidade a granel para os diferentes cenários de

Todos os ensaios mostraram que as densidades de carga das misturas de carvão aumentaram após serem submetidas a vibração, revelando que a técnica de aumento de densidade das misturas de carvão via vibração é promissora.

Os ensaios com apenas uma vibração mostraram que um longo tempo de vibração não melhora significativamente 0 aumento da densidade. Os valores encontrados no gráfico mostram que valores de percentual de aumento de 20, 30,60 e 120 segundos se encontram em um patamar próximo, com variação de aumento entre $11 \%$ e $14 \%$, em uma média de $12 \%$. Conclui-se que, para vibrações acima de 20 segundos, o ganho de densidade é pequeno, caso haja. Analisando esse modelo aplicado a um forno real, haveria um pequeno aumento de tempo de processo produzindo um elevação de $12 \%$ da densidade. O vibrador industrial estaria praticamente envolto a mistura de carvões ainda fria, sofreria menos com ação agressiva da temperatura do forno quando comparado ao que ocorreria em outros cenários.

Quando realizado o procedimento com mais de uma inserção, o resultado foi uma tendência de aumentar mais a densidade a granel com o aumento do número de inserções, atingindo o valor de $30 \%$ de aumento de densidade a granel com quatro inserções. Um ponto desfavorável deste cenário seria o maior tempo de processo, que poderia ser prejudicial tanto à produtividade do carro alimentador quanto pelo desgaste do vibrador no interior do forno. Outro ponto que também afetaria o seu desgaste seria a maior exposição a altas temperaturas, já que durante grande parte do seu tempo de trabalho, o vibrador estaria parcialmente não envolto pelo carvão, sofrendo pelo mecanismo de transferência de calor por radiação das paredes do forno. Apesar dos pontos levantados, é importante ressaltar que, como esse cenário obteve o maior aumento de densidade que chegou a 30\% de aumento, além do ganho de produtividade, frações significativas da carga de carvão HCC poderiam ser substituídas por carvões soft, acarretando uma redução significativa de custo.

O terceiro cenário apresenta uma alternativa intermediária entre os dois procedimentos desenvolvidos. O aumento de densidade em $20 \%$ já configura um ganho significativo para o processo. Apesar de estar exposto ao mecanismo de aquecimento por radiação, o tempo de exposição do vibrador no terceiro cenário quando comparado ao segundo é $35 \%$ menor, sendo esperado que sofra menos desgaste. A construção de uma nova "boca de carga" exclusiva para a inserção do vibrador, intermediárias às bocas de cargas existentes, poderia viabilizar esse cenário sem redução da taxa de alimentação e com pouco aumento do tempo de processo uma vez que a vibração é realizada concomitantemente à alimentação do carvão. 


\section{CONCLUSÃO}

A inovação proposta e verificada de aumento de densidade da mistura de carvões por vibração se confirmou. $\mathrm{O}$ aumento de densidade de carga chegou a atingir patamares de $30 \%$. Com um aumento de densidade dessa magnitude é possível substituir um percentual significativo de carvões $H C C$ por carvões soft, propiciando aumento de produtividade da coqueria e redução do custo da mistura de carvões. Comparada a outros métodos, a vibração foi superior aà Briquetagem Parcial, que aumenta em até $15 \%$ a densidade de carga, porém inferior ao Stamp Charging que consegue aumentos superiores a $50 \%$.

A simples exposição ao maior tempo de vibração não aumenta significativamente a densidade das misturas de carvões. Sucessivas inserções na carga devem ser realizadas para atingir o valor de $30 \%$ de aumento de densidade à granel.

\section{Agradecimentos}

Agradecimento ao CNPq, à REDEMAT, à Universidade Federal de Ouro Preto e à Gerdau Açominas.

\section{REFERÊNCIAS}

1 ANNUAL BOOK OF ASTM STANDARS - American Society for Metal, Philadelfia, USA.

2 SILVA, G.L.R. - Otimização da Mistura de Carvões na Produção de Coque Metalúrgico. [Dissertação de Mestrado] Pós-Graduação da Rede Temática em Engenharia dos Materiais, Universidade Federal de Ouro Preto. Ouro Preto, 2011.

3 LEIBROCK, K.; PETAK, H. - Coke Production from Low-Coking Coal Blends by Densification. Fuel Processing Technology. Elsevier Scientific Publishing Company. 1983;7: 91-107

4 AMORIM, O. V. - Estudo para o uso de matérias-primas em misturas de carvões para obtenção de coque com características requeridas em Altos-fornos de alta produtividade; [Dissertação de Mestrado] Curso de Pós-Graduação em engenharia Metalúrgica e de Minas da Universidade Federal de Minas Gerais. Belo Horizonte, 2005.

5 Madias, J.; - "Non-recovery/Heat-recovery Cokemaking - A review of recent developments, Acero Latinoamericano, 2010, 36-44.

6 João Batista Assis; Jorge Luiz Brescia Murta; Paulo Santos Assis; Ronaldo Sampaio Santos; Inventores. Processo para a elevação da densidade da mistura de carvões a coqueificar. Brasil PI 120623487. 18 de julho de 2012.

7 SILVA, J. M. - Estudo do Fluxo de Material Fragmentado na Mineração Subterrânea, com o Uso de Modelos Físicos. [Dissertação de Mestrado] Pós-Graduação em Engenharia Metalúrgica e de Minas, Belo Horizonte, 2005. 